\title{
Special issue on Efficient video coding and beyond
}

\author{
Mikhail K. Tchobanou' ${ }^{1}$ - Bin-Da Liu ${ }^{2}$
}

Published online: 11 April 2016

(C) Springer Science+Business Media New York 2016

The broad range of application areas for multi-dimensional signal processing systems and the need to tackle fundamental unsolved mathematical problems within this area provide strong motivation for further development of the underlying mathematical tools. Faster networks and more efficient video compression have enabled previously infeasible applications. The demand for higher-resolution content has further raised video traffic, to the point where, today, it occupies more and more of transmitted data in fixed and mobile networks. High Efficiency Video Coding (HEVC) was adopted as a new video coding standard in early 2013. Even though it has been reported that HEVC provided a bit rate reduction of about $50 \%$ at the same subjective quality when compared to advanced video coding (H.264/AVC), there is a need of further substantial improvement in its performance.

Motivated by these facts, this Special Issue aims at providing an overview of several recent developments in the wide area of (image) and video compression. The contributions selected for this Special Issue clearly display the broad scope of multidimensional systems, from open mathematical questions to practical engineering problems, and emphasize its interdisciplinary nature.

The contributions can be grouped into two thematic areas.

Video coding applications The main goal is to find possible promising approaches to the problem of video encoding. We are at the beginning of newly posted video compression problem and try to identify important crossroads, make a choice and eventually arrive to understanding of what are the limitations of the current encoding formats, where the reserves are and what would be the steps to a new encoding technique capable to go beyond those limits.

$凶 \quad$ Mikhail K. Tchobanou cmk2@orc.ru

Bin-Da Liu bdliu@mail.ncku.edu.tw

1 National Research University "MPEI”, Moscow, Russia

2 National Cheng Kung University, Tainan, Taiwan 
The paper by Pin-Chen Kuo, Jhih-Ming Lin, Bin-Da Liu, and Jar-Ferr Yang proposed an inpainting-based depth-image-based rendering algorithm for virtual view synthesis. Simulation results show that the synthesized virtual images for the proposed DIBR system have better quality than those obtained using other works (about 4-5 dB improvement). The parallel computation reduced runtime by 51 times and 25 times for generating nine and two views, respectively, compared to a single threaded approach. The method is realized on the compute unified device architecture parallel computing platform which runs on a graphics processing unit.

Chih-Wen Wang, Wei-Sheng Yang, Jyh-Horng Jeng and Jer-Guang Hsieh apply data reduction to images to achieve image compression. They propose a multi-stage clustering method to partition the data into groups such that individuals of the same group are homogeneous and vice versa. The method can effectively increase image quality and provides better visual effect. The proposed method effectively removes the coding redundancy and increases the number of principal components in some clusters in order to improve the reconstructed effect of certain clusters with complex structures.

Nick Petrovsky, Andrej Stankevich and Alexander Petrovsky proposed computational schemes that maintain losslessness for paraunitary filter banks regardless of their coefficient quantization. Quaternion multiplier lifting scheme based on the 2D CORDIC algorithm is the structural decision for the lossless digital signal processing. Lifting allows for making multiplications invertible. The 2D CORDIC engine using sparse iterations and asynchronous pipeline processor architecture based on the embedded CORDIC engine as stage of processor is reported. This approach applies to very practical filter banks, which are essential for image and video processing.

In the paper proposed by Alexander Zemliachenko, Vladimir Lukin, Nikolay Ponomarenko, Karen Egiazarian and Jaakko Astola it is demonstrated that there exist several coders for which the number of iterations can be essentially decreased using a reasonable selection of the starting value and the variation interval for the parameter controlling compression (PCC). PCC values attained at the end of the iterative procedure may heavily depend upon the coder used and the complexity of the image. Similarly, the compression ratio also considerably depends on the above factors. The authors show that for some modern coders that take HVS into consideration it is possible to give practical recommendations on setting a fixed PCC to provide a desired visual quality in a non-iterative manner.

Evgeny Petrov, Elena Medvedeva, Natalia Kharina and Mikhail Tchobanou present an intra-frame compression algorithm which allows 2-3 times increase in processing speed for comparable compression ratios. Nonlinear filtering algorithm of video (distorted during transmission on a radio channel in terms of interference) allows effective video restoring even for negative signal/noise ratio unlike the known methods. A distinctive feature of this algorithm is ease of implementation with minimal computing resources. Qiuwen Zhang, Huawen Chang, Qinggang Wu and Yong Gan propose a fast motion estimation/disparity estimation (ME/DE) algorithm based on inter-view and spatial correlations to reduce $3 \mathrm{D}-\mathrm{HEVC}$ computational complexity. Due to high correlation among the coding information from inter-view prediction, and due to spatially neighboring treeblocks have similar coding information, one can determine ME search range and skip some specific ME and DE rarely used in the previously coded view frames and spatially neighboring coding unit. Experimental results demonstrate that the proposed algorithm can significantly reduce computational complexity of 3D-HEVC encoding while maintaining almost the same rate-distortion performance. 
Video coding and human visual system Distortions perceived by the human being are not always captured by MSE/SNR/PSNR, because these metrics operate on a pixelby-pixel basis without considering the signal content, the viewing condition and the characteristics of the Human Visual System (HVS). These problems make the design of a better objective quality metric necessary, and progresses in recent vision research provide us with guidance to achieve this goal.

Michal Joachimiak, Miska M. Hannuksela and Moncef Gabbouj present a new depth encoding method called view upsampling optimization. In a mixed resolution $3 \mathrm{D}$ video stream, a subset of views is coded at reduced resolution. A novel depth distortion metric based on the performance of the depth-based super resolution is presented. An improved depth-based super resolution method that uses view synthesis quality mapping is used for upsampling of low resolution views. The simulations, performed with the recently standardized MVC+D encoder, show that the proposed solution outperforms the anchor MVC+D coding scheme by $14.5 \%$ of $\mathrm{dBR}$ on average for the total coded bitrate and by $17 \%$ of dBR on average for the synthesized views.

The paper by Minglei Tong, Zhouye Gu, Nam Ling and Junjie Yang propose to apply a technique of human centered perceptual computation to improve video coding in the region of human centered perception. By applying the proposed human perceptual framework in H.264/AVC video coding, improved perceptual quality of reconstructed video is achieved. The results show that the proposed algorithm can improve the visual quality of ROI by about $1.01 \mathrm{~dB}$ while preserving coding efficiency

We would like to thank all the authors for their contributions, the reviewers for their valuable suggestions, and the Editor-in-Chief while preparing this Special Issue, Zhiping Lin, for his kind help, valuable support and encouragement. 